\title{
Factores de riesgo de lesión de las arterias coronarias en niños con enfermedad de Kawasaki
}

Risk factors for coronary artery lesions in children with Kawasaki disease

\author{
Junyan Duan, Lic. en Medicina, ${ }^{a}$ Huanhuan Jiang, Lic. en Medicina, ${ }^{a} y$ \\ Ming Lu, Magister en Medicina ${ }^{b}$
}

\section{RESUMEN}

Introducción. La enfermedad de Kawasaki (EK) es una vasculitis sistémica inespecífica que suele presentarse en los niños; la lesión de las arterias coronarias (LAC) es la complicación más grave. Objetivos. Nuestro objetivo fue investigar los factores de riesgo de LAC en niños con EK.

Materiales y métodos. Se incluyó a niños con EK según los criterios diagnósticos, hospitalizados entre enero de 2014 y diciembre de 2017. Se realizaron análisis univariado y multivariado de regresión logística para investigar las relaciones entre LAC y género, edad, diagnóstico clínico, velocidad de sedimentación globular (VSG), recuento de trombocitos, concentración de hemoglobina, concentración de proteína C-reactiva, recuento de leucocitos, momento de inicio de la administración de inmunoglobulina intravenosa (IgIV) y duración de la fiebre.

Resultados. Se dividió a los 982 niños con EK en un grupo con LAC $(\mathrm{n}=104)$ y otro sin LAC $(\mathrm{n}=878)$, según una ecocardiografía Doppler color. La tasa de incidencia de LAC fue del 10,6 \% (104/982). En el análisis univariado, se observó una diferencia significativa entre ambos grupos en cuanto al género, la VSG, el recuento de trombocitos, el momento de inicio de la administración de IgIV y la duración de la fiebre $(p<0,05)$. Según el análisis multivariado de regresión logística, el sexo masculino, una VSG elevada y la administración tardía de IgIV fueron factores de riesgo independientes de EK complicada con LAC.

Conclusiones. El sexo masculino, una VSG elevada y la administración tardía de IgIV fueron factores de riesgo independientes de EK complicada con LAC.

Palabras clave: enfermedad de Kawasaki, arteria coronaria, regresión logística, factores de riesgo.

http:/ / dx.doi.org/10.5546/aap.2020.327

Texto completo en inglés:

http: / / dx.doi.org/10.5546/ aap.2020.eng.327

Cómo citar: Duan J, Jiang H, Lu M. Factores de riesgo de lesión de las arterias coronarias en niños con enfermedad de Kawasaki. Arch Argent Pediatr 2020;118(5):327-331.

\section{INTRODUCCIÓN}

La enfermedad de Kawasaki (EK) es una vasculitis sistémica inespecífica que suele presentarse en los niños, y la lesión de las arterias coronarias (LAC) es la complicación más grave, con una tasa de incidencia del $20 \%$ al $35 \%{ }^{1}$ Según la evidencia acumulada, las LAC provocadas por la EK persisten durante muchos años y pueden causar cardiopatía coronaria en la adultez..$^{2-4}$ Las LAC han remplazado a la fiebre reumática como una de las principales causas de cardiopatía adquirida en los niños. En los últimos años, el diagnóstico y el tratamiento tempranos de la EK siguen siendo difíciles debido a que se desconoce su etiología. ${ }^{5} \mathrm{La}$ EK puede provocar LAC, las cuales pueden causar estenosis e infarto trombótico, infarto de miocardio e incluso muerte súbita. Por lo tanto, es fundamental determinar los factores de riesgo lo antes posible y tomar medidas eficaces, razonables y oportunas para reducir la incidencia y la tasa de mortalidad de la EK. El objetivo de este estudio fue investigar los factores de riesgo de LAC en niños con EK.

\section{MATERIALES Y MÉTODOS}

Se realizó un estudio unicéntrico con una muestra grande para analizar los datos clínicos de niños con EK.

\section{Datos clínicos iniciales}

Se seleccionó a los niños con EK hospitalizados entre enero de 2014 y diciembre de 2017. Este estudio fue aprobado por el comité de ética de nuestro hospital el 4 de enero de 2014 (aprobación n. ${ }^{\circ}$ AHXMU 2014-01002), 
y se obtuvo el consentimiento informado firmado por los tutores de todos los participantes.

\section{Criterios diagnósticos}

Se diagnosticó EK según las pautas de diagnóstico y tratamiento de la EK propuestas por la Academia Estadounidense de Pediatría y la Asociación Estadounidense del Corazón en 2004 . $^{6}$ Se les diagnosticó EK a los niños que tuvieron fiebre (entre 39 y $40{ }^{\circ} \mathrm{C}$ ) durante más de 5 días y 4 de estas 5 manifestaciones clínicas: 1) cambios en las cuatro extremidades: eritema palmoplantar durante la fase aguda, edema indurado de manos y pies, descamación de los pulpejos de los dedos durante la fase de convalecencia; 2) exantema polimorfo; 3) hiperemia conjuntival bilateral; 4) labios eritomatosos y fisurados, congestión difusa de la mucosa oral y papilas prominentes (lengua aframbuesada); 5) adenopatía cervical. Si un niño tenía fiebre (entre 39 y $40{ }^{\circ} \mathrm{C}$ ) durante más de 5 días y no presentaba 4 de las 5 manifestaciones clínicas anteriores, pero en la ecocardiografía Doppler color se observaba dilatación coronaria o enfermedades febriles como la escarlatina, se le diagnosticó EK incompleta luego de descartar el síndrome de hipersensibilidad a medicamentos, el síndrome de Stevens-Johnson, el síndrome del choque tóxico, una infección por adenovirus o la infección por el virus de Epstein-Barr.

Según la fórmula del valor normal de la arteria coronaria corregida por superficie corporal que desarrollaron de Zorzi y cols., ${ }^{7}$ el valor Z se calculó de esta manera: $Z=$ (valor de la observación valor normal medio)/desviación estándar. Se determinó la presencia de LAC cuando el valor Z del diámetro interno de la arteria descendente anterior izquierda o de la arteria coronaria izquierda o derecha superaba 2,5. Se definió como dilatación en las arterias coronarias un puntaje de $\mathrm{Z}$ superior a 2,5 y un diámetro interno de la arteria coronaria superior o igual a $4 \mathrm{~mm}$. Aneurisma coronario: aneurisma coronario pequeño (diámetro interno de la arteria coronaria: $>4 \mathrm{~mm} \mathrm{y}<5 \mathrm{~mm}$ ), aneurisma coronario mediano (diámetro interno: entre 5 y $8 \mathrm{~mm}$ ) y aneurisma coronario gigante (diámetro interno: $>8 \mathrm{~mm}$ ). ${ }^{8}$ La superficie corporal se calculó de la siguiente forma: ${ }^{9}$ si el peso corporal era inferior a $30 \mathrm{~kg}$, superficie corporal $\left(\mathrm{m}^{2}\right)=$ masa corporal $(\mathrm{kg})$ $\times 0,035\left(\mathrm{~m}^{2} / \mathrm{kg}\right)+0,1\left(\mathrm{~m}^{2}\right)$; si el peso corporal era de entre 30 y $50 \mathrm{~kg}$, la superficie corporal se incrementó $0,1 \mathrm{~m}^{2}$ por cada $5 \mathrm{~kg}$ de peso adicionales.

\section{Análisis estadístico}

El análisis estadístico de todos los datos se realizó con el programa SPSS 17.0. Para las comparaciones de los datos numéricos entre los grupos, se utilizó la prueba $\chi^{2}$, y los datos cuantitativos con distribución normal se expresaron como media \pm desviación estándar $(x \pm D E)$. Se realizaron análisis univariado y multivariado de regresión logística para esclarecer las relaciones entre las LAC

TABla 1. Análisis univariado de los factores de riesgo de LAC en niños con EK

\begin{tabular}{|c|c|c|c|c|}
\hline & $\begin{array}{c}\text { Grupo con LAC } \\
(\mathrm{n}=104)\end{array}$ & $\begin{array}{l}\text { Grupo sin LAC } \\
\quad(\mathrm{n}=878)\end{array}$ & $t / \chi^{2}$ & $p$ \\
\hline \multicolumn{5}{|l|}{$\overline{\text { Sexo }}$} \\
\hline Masculino & 80 & 518 & 12,547 & 0,000 \\
\hline Femenino & 24 & 360 & & \\
\hline Edad (meses) & $30,47 \pm 5,48$ & $31,38 \pm 5,78$ & 1,526 & 0,127 \\
\hline \multicolumn{5}{|l|}{ Diagnóstico clínico } \\
\hline EK típica & 60 & 576 & 2,550 & 0,110 \\
\hline EK incompleta & 44 & 302 & & \\
\hline $\operatorname{VSG}(\mathrm{mm} / \mathrm{h})$ & $69,62 \pm 9,18$ & $82,71 \pm 8,94$ & 14,079 & 0,000 \\
\hline Recuento de trombocitos $\left(\times 10^{9} / 1\right)$ & $352,68 \pm 23,48$ & $432,58 \pm 24,17$ & 31,972 & 0,000 \\
\hline Concentración de hemoglobina (g/l) & $9,36 \pm 1,95$ & $9,67 \pm 1,54$ & 1,882 & 0,060 \\
\hline PCR $(\mathrm{mg} / 1)$ & $69,23 \pm 6,15$ & $68,89 \pm 6,35$ & 0,518 & 0,605 \\
\hline Recuento de leucocitos $\left(\times 10^{9} / 1\right)$ & $15,46 \pm 2,11$ & $15,24 \pm 2,32$ & 0,923 & 0,356 \\
\hline Momento de inicio de la administración de IgIV (d) & $6,7 \pm 1,5$ & $9,5 \pm 1,6$ & 16,984 & 0,000 \\
\hline Duración de la fiebre (d) & $8,3 \pm 1,2$ & $9,7 \pm 1,3$ & 10,466 & 0,000 \\
\hline
\end{tabular}

LAC: lesión de las arterias coronarias; EK: enfermedad de Kawasaki; VSG: velocidad de sedimentación globular; PCR: proteína C-reactiva. 
y el género, la edad, el diagnóstico clínico, la velocidad de sedimentación globular (VSG), el recuento de trombocitos, la concentración de hemoglobina, la concentración de proteína C-reactiva (PCR), el recuento de leucocitos, el momento de inicio de la administración de IgIV y la duración de la fiebre. El análisis univariado se realizó con la prueba t en muestras independientes, y se sometió a las variables con significación estadística a un análisis multivariado de regresión logística. Un valor de $p<0,05$ se consideró significativo en términos estadísticos.

\section{RESULTADOS}

\section{Datos clínicos iniciales}

De los 982 pacientes con EK, 598 eran niños y 384, niñas. La edad de aparición de $64(6,5 \%)$, $814(82,9 \%)$ y $104(10,6 \%)$ casos era $\leq 6$ meses, entre 6 meses y 5 años y $>5$ años, respectivamente. En total, hubo 634 casos $(64,6 \%)$ de EK típica, y los otros 348 casos $(35,4 \%)$ correspondían a EK incompleta. Los niños con EK incluidos se dividieron en un grupo con LAC $(n=104)$ y un grupo $\sin \operatorname{LAC}(\mathrm{n}=878)$, según una ecocardiografía Doppler color; todos ellos recibieron el diagnóstico por primera vez. La tasa de incidencia de LAC fue del 10,6 \% (104/982).

\section{Análisis univariado de los factores de riesgo de LAC en niños con EK}

En el análisis univariado, se observó una diferencia significativa entre ambos grupos en cuanto al género, la VSG, el recuento de trombocitos, el momento de inicio de la administración de IgIV y la duración de la fiebre $(p<0,05)$ (Tabla 1).

\section{Análisis multivariado de regresión logística de los factores de riesgo de LAC en niños con EK}

Según el análisis multivariado de regresión logística, el sexo masculino, una VSG elevada y la administración tardía de IgIV fueron factores de riesgo independientes de EK complicada con LAC (Tabla 2).

\section{DISCUSIÓN}

Dado que se trata de una vasculitis inmunitaria autolimitada que afecta los vasos pequeños y medianos, ${ }^{10}$ la EK suele provocar la dilatación de las arterias coronarias o la formación de aneurismas gigantes, y conlleva posibles riesgos de infarto de miocardio e incluso de muerte súbita de origen cardíaco. ${ }^{11}$ Por este motivo, el diagnóstico temprano y el tratamiento oportuno de la EK han sido objeto de una atención generalizada, y los factores de riesgo de EK complicada con LAC también se han convertido en una prioridad para los pediatras.

Se informó que la edad inferior a 1 año o superior a 8 años, el sexo masculino, la VSG, la concentración de PCR, el recuento de leucocitos, el recuento de trombocitos, la concentración de albúmina, la administración tardía de IgIV en dosis bajas y la recurrencia de EK son factores de riesgo de LAC. ${ }^{12}$ En este estudio, el sexo (masculino) fue un factor de riesgo independiente de LAC. Además, es posible que los varones con EK tengan una predisposición genética específica a las LAC. Por lo tanto, la EK se puede definir como una respuesta autoinmunitaria que tiene una predisposición genética y es ocasionada por estímulos externos. Además, el riesgo elevado de LAC en poblaciones específicas sugiere que la predisposición genética tiene una función importante en la patogenia de la EK. ${ }^{13}$

En este estudio, la edad, la duración de la fiebre, la concentración de PCR o el recuento de leucocitos de los niños con EK no se correlacionaron significativamente con las LAC. Probablemente, en el $80 \%$ de los niños con EK, la enfermedad comience entre los 6 meses y los 4 años. ${ }^{14}$ En niños menores de 1 año o mayores de 5 años,

TABLA 2. Análisis multivariado de regresión logística de los factores de riesgo de LAC en niños con EK

\begin{tabular}{|c|c|c|c|c|c|}
\hline Factor & $\beta$ & Wald & OR & IC del $95 \%$ & Valor $p$ \\
\hline Sexo & $-1,009$ & 7,267 & 0,364 & $0,175-0,756$ & 0,006 \\
\hline VSG & 0,019 & 11,243 & 1,019 & $1,004-1,345$ & 0,001 \\
\hline \multicolumn{6}{|l|}{ Momento de inicio de la } \\
\hline administración de IgIV & 0,257 & 28,895 & 1,287 & $1,145-1,458$ & 0,001 \\
\hline Recuento de trombocitos & 0,001 & 2,798 & 1,004 & $0,982-1,155$ & 0,879 \\
\hline Duración de la fiebre & 0,004 & 0,743 & 1,043 & $0,942-1,156$ & 0,458 \\
\hline
\end{tabular}

LAC: lesión de las arterias coronarias; EK: enfermedad de Kawasaki; VSG: velocidad de sedimentación globular. 
las manifestaciones clínicas de la EK suelen ser atípicas, por lo que los médicos no pueden diagnosticar la enfermedad en las primeras etapas $y$, en consecuencia, los niños no reciben el tratamiento con IgIV de forma oportuna; esto ocasiona un aumento significativo en la tasa de incidencia de las LAC. ${ }^{15}$ La EK provoca una lesión endotelial vascular al activar las células inmunitarias y potenciar la respuesta inmunitaria por razones desconocidas. ${ }^{16} \mathrm{La}$ IgIV puede aliviar la inflamación y la respuesta inmunitaria de forma significativa, bloquear los receptores Fc en la superficie de los trombocitos y tener efectos antinflamatorios inespecíficos, lo que mitiga las LAC. ${ }^{17,18}$ Además, la administración temprana ( $<10$ días) de IgIV puede reducir el riesgo de LAC en EK de forma significativa, lo que coincide con los resultados de este estudio. ${ }^{19}$

La PCR y la VSG son marcadores de laboratorio ampliamente reconocidos de respuesta inflamatoria, y ambos se encuentran elevados en las primeras etapas de la EK, con valores diagnósticos altos. Kawasaki fue el primero en informar en 1967 que alrededor del $36 \%$ de los niños con EK tenían cambios desincronizados en la VSG y la PCR. ${ }^{20}$ Bray y cols. ${ }^{21}$ también observaron que aproximadamente el $44 \%$ de los niños con EK tenían manifestaciones similares. La PCR es una proteína reactiva de fase aguda que aumenta entre 4 y 6 horas después de la estimulación inflamatoria, llega a su concentración máxima entre 36 y 50 horas después de la estimulación y disminuye entre 3 y 7 días luego de que finaliza la estimulación, mientras que la VSG se determina según la velocidad de disminución de la masa eritrocitaria en el plasma sanguíneo, que se ve afectada por el tamaño, la forma y la cantidad de eritrocitos y la concentración de inmunoglobulina. La VSG aumenta lentamente y regresa al valor inicial más lentamente, una vez que los factores inflamatorios están ausentes. La VSG demora más que la PCR en alcanzar el valor máximo. Por lo tanto, el aumento de la PCR y la VSG en los niños con EK es desincronizado. Además, una VSG elevada sugiere que la EK se encuentra en una fase avanzada, la repuesta inflamatoria dura mucho tiempo y la arteria coronaria estuvo expuesta a factores inflamatorios de forma prolongada, lo que podría ocasionar lesiones vasculares. Por lo tanto, un aumento en la VSG puede reflejar, en cierta medida, el daño que ocasionó la respuesta inflamatoria en la arteria coronaria. ${ }^{22}$ Cabe destacar que la VSG es sensible a la globulina plasmática. En consecuencia, se debe utilizar la VSG antes de la administración de IgIV como punto de partida para evaluar la gravedad de la EK. En 2005, Gómez de Diego y cols. utilizaron una coronariografía mediante tomografía computarizada (TC) multicorte para evaluar la anatomía coronaria. ${ }^{23}$ Recientemente, Goh y cols. señalaron que la coronariografía mediante TC constituía una opción atractiva para usar en niños con EK, en lugar de la coronariografía invasiva. ${ }^{24}$ Además, Jrad y cols. observaron que la coronariografía con TC era superior para detectar lesiones coronarias fusiformes, así como las ramas distales y posteriores, en la EK. No obstante, la ecocardiografía transtorácica seguía siendo el estudio de elección debido a sus características de radiación y disponibilidad. ${ }^{25}$

En este estudio, el sexo masculino, una VSG elevada y la administración tardía de IgIV fueron factores de riesgo independientes de EK complicada con LAC. Asimismo, la cantidad de índices de observación que se incluyeron fue limitada. Por lo tanto, se requieren estudios exhaustivos adicionales para hallar índices de predicción más específicos, con el objetivo de ofrecer una base teórica para el diagnóstico y el tratamiento tempranos de la EK.

\section{CONCLUSIONES}

A modo de resumen, el sexo masculino, una VSG elevada y la administración tardía de IgIV fueron factores de riesgo independientes en niños con EK complicada por LAC.

\section{REFERENCIAS}

1. PatelRM, ShulmanST. Kawasaki disease: a comprehensive review of treatment options. J Clin Pharm Ther. 2015;

2. Kim MK, Song MS, Kim GB. Factors Predicting Resistance to Intravenous Immunoglobulin Treatment and Coronary Artery Lesion in Patients with Kawasaki Disease: Analysis of the Korean Nationwide Multicenter Survey from 2012 to 2014. Korean Circ J. 2018; 48(1):71-9.

3. Xie T, Wang Y, Fu S, Wang W, et al. Predictors for intravenous immunoglobulin resistance and coronary artery lesions in Kawasaki disease. Pediatr Rheumatol Online J. 2017; 15(1):17.

4. Lee HY, Song MS. Predictive factors of resistance to intravenous immunoglobulin and coronary artery lesions in Kawasaki disease. Korean J Pediatr. 2016; 59(12):477-82.

5. Li XQ, Kuang L, Wu J, Hu C, et al. [Progress in treatment of Kawasaki disease]. J Modern Clin Med. 2016; 42:328-30.

6. Newburger JW, Takahashi M, Gerber MA, Gewitz MH, et al. Diagnosis, treatment, and long-term management of Kawasaki disease: a statement for health professionals from the Committee on Rheumatic Fever, Endocarditis, and Kawasaki Disease, Council on Cardiovascular Disease in the Young, American Heart Association. Pediatrics. 2004; 114(6):1708-33.

7. deZorzi A, ColanSD, Gauvreau K, Baker AL, et al. Coronary 
artery dimensions may be misclassified as normal in Kawasaki disease. J Pediatr. 1998; 133(2):254-8.

8. McCrindle BW, Rowley AH, Newburger JW, Burns JC, et al. Diagnosis, Treatment, and Long-Term Management of Kawasaki Disease: A Scientific Statement for Health Professionals from the American Heart Association. Circulation. 2017; 135(17):e927-99.

9. Yang HM, Du ZD, Fu PP. Clinical features of recurrent Kawasaki disease and its risk factors. Eur J Pediatr. 2013; 172(12):1641-7.

10. Mortazavi SH, Amin R, Alyasin S, Kashef S, et al. Downregulation of TLR2, 3, 9 and Signaling Mediators, MyD88 and TRIF, Gene Transcript Levels in Patients with Kawasaki Disease Treated with IVIG. Iran J Allergy Asthma Immunol. 2015; 14(2):188-97.

11. Liu MY, Liu HM, Wu CH, Chang $\mathrm{CH}$, et al. Risk factors and implications of progressive coronary dilatation in children with Kawasaki disease. BMC Pediatr. 2017; 17(1):139.

12. Giannouli G, Tzoum aka-B akoula C, Kops id as I, Papadogeorgou P, et al. Epidemiology and Risk Factors for Coronary Artery Abnormalities in Children with Complete and Incomplete Kawasaki Disease During a 10-Year Period. Pediatr Cardiol. 2013; 34(6):1476-81.

13. Yoon KL. Update of genetic susceptibility in patients with Kawasaki disease. Korean J Pediatr. 2015; 58(3):84-8.

14. Greco A, De Virgilio A, Rizzo MI, Tombolini M, et al. Kawasaki disease: an evolving paradigm. Autoimmun Rev. 2015; 14(8):703-9.

15. Yeom JS, Woo HO, Park JS, Park ES, et al. Kawasaki disease in infants. Korean J Pediatr. 2013; 56(9):377-82.

16. Baraona F, Valente AM, Porayette P, Pluchinotta FR, Sanders SP. Coronary Arteries in Childhood Heart Disease: Implications for Management of Young Adults. J Clin Exp Cardiolog. 2012; (Suppl 8):006.
17. Galeotti C, Bayry J, Kone-Paut I, Kaveri SV. Kawasaki disease: Aetiopathogenesis and therapeutic utility of intravenous immunoglobulin. Autoimmun Rev. 2010; 9(6):441-8.

18. Kaneko K, Takahashi M, Yoshimura K, Kitao T, et al. Intravenous Immunoglobulin Counteracts OxidativeStress in Kawasaki Disease. Pediatr Cardiol. 2012; 33(7):1086-8.

19. Qiu H, He Y, Rong X, Ren $Y$, et al. Delayed intravenous immunoglobulin treatment increased the risk of coronary artery lesions in children with Kawasaki disease at different status. Postgrad Med. 2018; 130(4):442-7.

20. Zhu FH, Ang JY. The clinical diagnosis and management of Kawasaki disease: A review and update. Curr Infect Dis Rep. 2016; 18(10):32.

21. Bray C, Bell LN, Liang H, Haykal R, et al. Erythrocyte Sedimentation Rate and C-reactive Protein Measurements and Their Relevance in Clinical Medicine. WMJ. 2016; 115(6):317-21.

22. Chen J, Liu Y, Liu W, Wu Z. A meta-analysis of the biomarkers associated with coronary artery lesions secondary to Kawasaki disease in Chinese children. J Huazhong Univ Sci Technolog Med Sci. 2011; 31(5):705.

23. Gómez de Diego JJ, García Fernández MA, Sales Sales JR. Enfermedad de Kawasaki estudiada mediante TC multidetectora. Rev Esp Cardiol. 2005; 58(10):1224-5.

24. Goh YG, Ong CC, Tan G, Liang CR, et al. Coronary manifestations of Kawasaki disease in computed tomography coronary angiography. J Cardiovasc Comput Tomogr. 2018; 12(4):275-80.

25. Jrad M, Ben Salem F, Barhoumi C, Lassoued F, et al. The Role of Computed Tomography Coronary Angiography in Kawasaki Disease: Comparison with Transthoracic Echocardiography in a 25-Case Retrospective Study. Pediatr Cardiol. 2019; 40(2):265-75. 